Trauma Berufskrankh $2003 \cdot 5$ [Suppl 2]: S328-S331 DOI 10.1007/s10039-002-0659-y

Septische Chirurgie

Christian Hierholzer · Thomas Mückley · Martin H. Kirschner · Gunther O. Hofmann

Abteilung für Septische und Wiederherstellungschirurgie, BG-Unfallklinik Murnau

\title{
Revisionsendoprothetik am Kniegelenk beim Infekt
}

ie Infektion einer Kniegelenkendoprothese stellt eine der gravierendsten Komplikationen im postoperativen Verlauf nach Implantation dar und beinhaltet eine erhebliche Morbidität. Die Infektion reduziert die Langzeitprognose der Prothese und kann bei Nichtbeherrschen der Infektsituation zu einem Funktionsverlust des Gelenks führen und bis zur Gefährdung der Extremität reichen. Für die betroffenen Patienten bedeutet die Protheseninfektion nicht nur eine Verschlechterung der Lebensqualität, sondern u. U. auch den Verlust der sozialen Unabhängigkeit.

In der Gelenkendoprothetik stehen Infektionen von Knieprothesen mit einer Inzidenz von 2,6-6\% in der Häufigkeitsverteilung an erster Stelle [1]. Die Infektraten bei Hüftendoprothesen liegen mit $1,3 \%$ deutlich niedriger [12]. Die unterschiedliche Weichteildeckung spielt kausal eine wesentliche Rolle [13]. Der Infekt ist v. a. im so genannten Interface lokalisiert, also der Grenzschicht zwischen Knochen und Prothese. Hier finden Keime gute Adhäsionsmöglichkeiten, und die Zone ist für antimikrobielle Substanzen kaum zugänglich [2]. Nach dem zeitlichen Auftreten des Infekts nach Implantation der Endoprothese wird zwischen

- einem Frühinfekt und

- einem Spätinfekt

unterschieden. Der Frühinfekt tritt definitionsgemäß innerhalb der ersten
8 Wochen auf, der Spätinfekt ist durch ein späteres Auftreten gekennzeichnet [7].

\section{Diagnostik}

Diagnostisch kommt der Klinik besondere Bedeutung zu. Jede Wundheilungsstörung, Weichteilschwellung und Wundsekretion müssen sorgfältig evaluiert werden [10]. Der Lockerungsschmerz kann Hinweis auf eine chronische Infektion sein $[6,12]$. In der Sonographie können Flüssigkeitsverhalte nachgewiesen und lokalisiert werden. Im Labor werden die Entzündungsparamenter Leukozytenzahl und CRP bestimmt. Hinweisend für das Vorliegen einer Infektsituation ist der erneute CRP-Anstieg nach regelrechtem postoperativem CRPAbfall. Der direkte mikrobiologische Keimnachweis ist die Grundlage der Diagnose.

In einer prospektiven Studie, die mit der Orthopädischen Klinik der TU München durchgeführt wird, wurde ein

๑) Springer-Verlag 2003

Prof.Dr.Dr. Gunther 0. Hofmann

Abteilung für Septische

und Wiederherstellungschirurgie,

BG-Unfallklinik Murnau,

Prof.-Küntscher-Straße 8,

82418 Murnau,

E-Mail: gunther.hofmann@bgu-murnau.de,

Phone: 08841-482451, Fax: 08841-482117 
C. Hierholzer · T. Mückley · M.H. Kirschner • G.O.Hofmann

\section{Treatment of infected total knee arthroplasty}

\section{Abstract}

Infection of total knee arthroplasty requires a complex revision strategy related to stage for successful treatment of the infection and preservation of the total knee prothesis. In the present study, an operative concept of programmed revisions by arthroscopic and arthrotomic techniques was evaluated in 34 patients suffering from infections of total knee arthroplasties. In 13 patients the infection was successfully treated with the prothesis left in place: 9 of these patients underwent arthroscopic revision and the other 4 , open revision and irigation. In 21 cases removal of the prothesis was necessary to control the infection. Following successful treatment of infection and negative bacterial cultures in three consecutive operations reconstruction was performed. In 14 cases twostage re-implantation of the total knee arthroplasties was performed after a 12-week interval. Six patients underwent arthrodesis due to problems of bone loss and soft tissue damage. In 1 case amputation of the lower extremity was necessary following vital systemic complications. These results demonstrate that infection of total knee arthroplasties can be successfully treated using a concept of repetitive arthroscopic and arthrotomic revisions. In cases of early infection the prothesis may be left in place. If removal of a prothesis is necessary we prefer a two-stage approach to re-implantation of the knee prothesis after a 12-week interval.

\section{Keywords}

Infection of total knee prothesis . Programmed revisions - Two-stage re-implantation operatives Konzept der programmierten Lavage evaluiert. Ziel war es, Infektionen von Knieendoprothesen durch kurz aufeinander folgende Revisionen zu sanieren und damit Endoprothesen dauerhaft zu erhalten.

\section{Material und Methode}

Seit 1996 wurden 34 Patienten mit Infektionen von Knieprothesen therapiert und ausgewertet. In der Pathogenese der Kniegelenkzerstörung fanden sich

- eine posttraumatische Gonarthrose bei 14 Patienten,

- eine idiopathische Gonarthrose bei 9 Patienten,

- eine chronische Polyarthritis bei 9 Patienten und

- eine Tumorerkrankung bei 2 Patienten.

Das Revisionsprotokoll (Abb. 1) sieht bei Frühinfekten die Durchführung von arthroskopisch assistierten Lavageeingriffen in 2- bis 4-tägigem Abstand vor. Kann mit den arthroskopischen Eingriffen keine Keimfreiheit erzielt werden, werden offene Revisionen durchgeführt. Spätinfektionen von Knieprothesen werden initial mit offenen Revisionen bis zur Keimfreiheit therapiert. Der Revisionseingriff umfasst
- ein radikales Débridement von in fiziertem und bradytrophem Gewebe,

- die Synovektomie,

- die Ausräumung von Defekten in der Markhöhle sowie

- die Resektion von avitalem Knochen.

Zur Ergänzung der bakteriologischen Diagnostik werden Gewebeproben entnommen.

Prothesenkomponenten müssen mechanisch gereinigt werden, um die von Bakterien, wie z. B. koagulasenegativen Staphylokokken, gebildete extrazelluläre Schleimsubstanz (ESS) zu entfernen. Diese Schicht schützt vor Phagozytose und antiseptischer Therapie [9].

Nach der Prothesenreinigung folgen Spülung, Jet-Lavage und Applikation eines lokalen Antiseptikums sowie Einbringen eines lokalen Antibiotikums über einen Trägerschwamm. Die Wunde wird mit Vakuumversiegelung verschlossen. Postoperativ erhalten die $\mathrm{Pa}$ tienten eine systemische, testgerechte Antibiose.

Bei Infektpersistenz ist der Prothesenausbau erforderlich. Zur temporären Fixierung wird ein Fixateur externe angelegt, die Weichteile werden saniert, bis ein stabiler Weichteilmantel vorliegt.

Nach Infektausheilung kann im Anschluss an ein 12-wöchiges Intervall die Reimplantation der Endoprothese erfol-

\section{Endoprotheseninfekt}

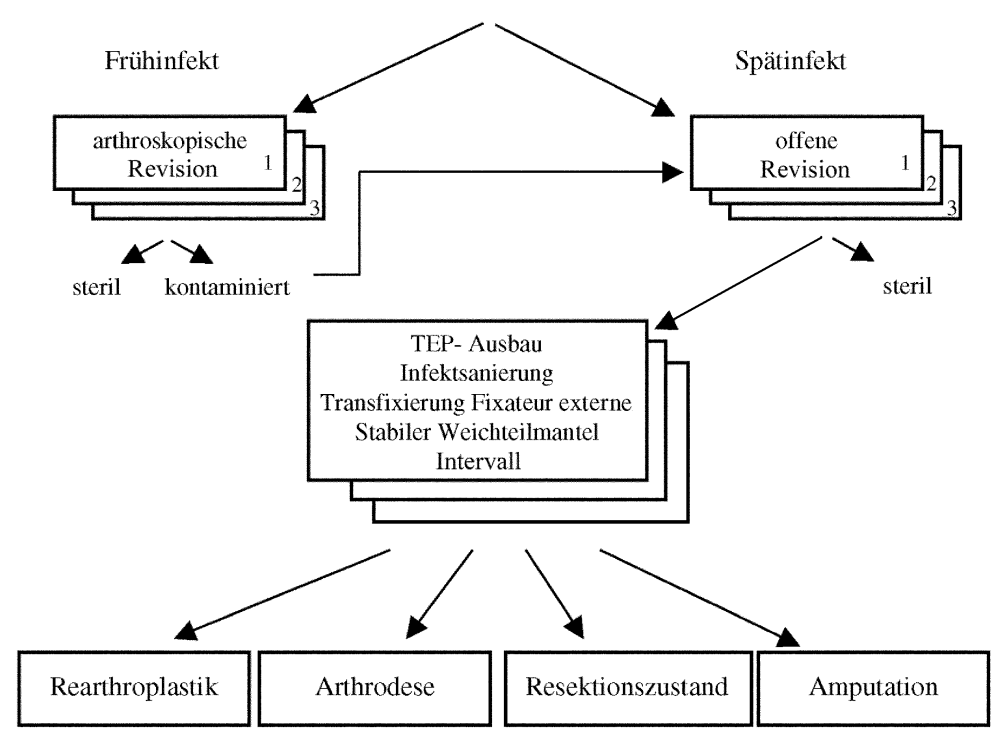

Abb. $1 \Delta$ Therapiekonzept der programmierte Revisionen bei Infektion einer Kniegelenkendoprothese 


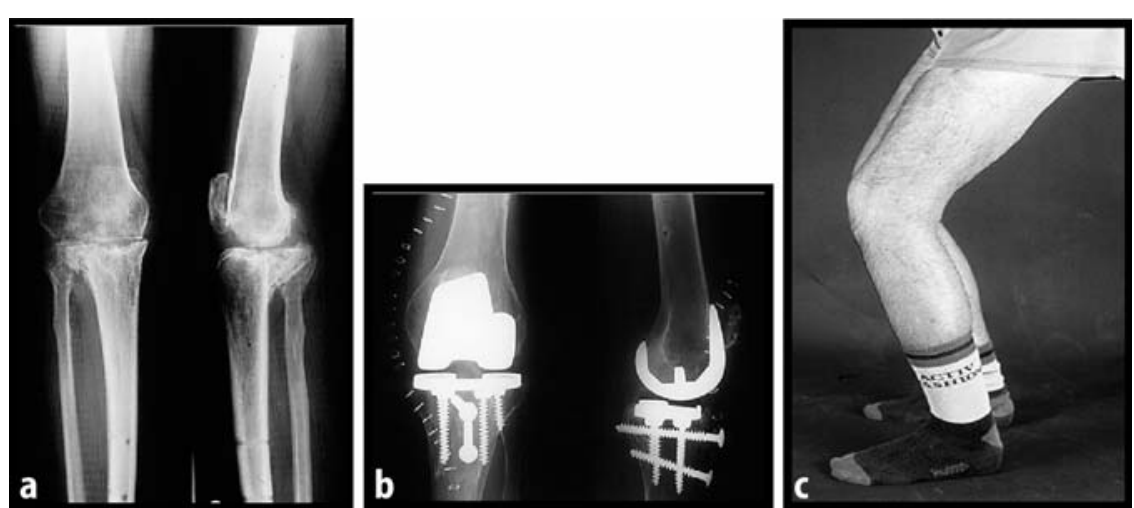

Abb. 2a-c $\Delta$ Revisionsendoprothetik am Kniegelenk. 65-jähriger Patient mit Zustand nach Infektion einer Kniegelenkendoprothese.Zweizeitiges Vorgehen: Entfernung der Prothese. Nach erfolgreicher Infektsanierung wurde bei ausreichendem knöchernem Fundament (a) und erhaltener Kollateralbandstabilität nach einem 12-wöchigen Intervall die Implantation einer nicht-gekoppelten, duokondylären Schlittenprothese durchgeführt $(b, c)$

gen. Voraussetzung ist ein ausreichendes knöchernes Fundament. Bei knöchernem Substanzdefekt kommt alternativ die Arthrodese in Frage. Der Resektionszustand oder die Amputation stellen therapeutische Ausnahmen dar.

\section{Ergebnisse}

Seit 1996 wurden 34 Patienten mit Protheseninfektionen therapiert. Das Patientenkollektiv setzte sich aus 19 Männern und 15 Frauen zusammen. Die Altersverteilung entsprach dem typischen Patientengut. Das mittlere Alter betrug 51,5 Jahre (19-81 Jahre).

Bei 13 der 34 Patienten konnte die Infektion unter Prothesenerhalt saniert werden, davon in 9 Fällen mit arthroskopischen Revisionen und in 4 Fällen mit offenen Revisionen. Bei 21 Patienten war die Prothesenentfernung zur Beherrschung des Infekts erforderlich.Zur Sanierung und Konditionierung der Weichteile wurde eine temporäre Fixierung mit Fixateur externe durchgeführt.

Im Anschluss an ein 12-wöchiges Intervall mit negativem bakteriologischem Befund und klinischer sowie laborchemischer Infektfreiheit konnte bei 14 Patienten die zweizeitige Reimplantation einer Prothese durchgeführt werden. Bei diesen Patienten lagen sanierte und stabile Weichteilverhältnisse, eine suffiziente Funktion des Streckapparats und ein ausreichendes Knochenfundament vor (Abb. 2). Bei 6 weiteren Patienten wurde eine Arthrodese durchgeführt, und bei 1 Patienten war die Ober- schenkelamputation aus vitaler Indika-

\section{Diskussion}

In der vorgelegten Studie wurde ein konsequentes und radikales Revisionsprogramm zur Sanierung von infizierten Kniegelenktotalprothesen evaluiert. Mit Hilfe von programmierten arthroskopischen und offenen Revisionen, die in kurz aufeinander folgenden Abständen durchgeführt wurden, konnten die Infektionen therapiert werden. Bei $38 \%$ der Patienten wurde die Infektsanierung unter Prothesenerhalt erzielt. Bei $42 \%$ wurde eine erfolgreiche Revisionsendoprothetik in einem zweizeitigen Vorgehen durchgeführt.

Die Ergebnisse zeigen, dass mit Hilfe des vorgestellten Revisionsprogramms sowohl eine Infektsanierung unter Prothesenerhalt möglich war als auch eine hohe Rate an Prothesenreimplantationen bei den Patienten erzielt werden konnte, bei denen der Prothesenausbau zur Infektsanierung erforderlich war. Im Literaturvergleich konnte damit v. a. die Rate an erfolgreicher Revisionsendoprothetik gesteigert werden $[7,11]$.

Der Sanierungserfolg hängt entscheidend vom zeitlichen Auftreten der Infektion ab. Die Frühinfekte, die innerhalb der ersten 6 Wochen nach Implantation der Endoprothese auftraten und in diesem Zeitintervall diagnostiziert wurden, heilten bei allen Patienten $(n=12)$ unter Prothesenerhalt aus. Bei tion erforderlich.
Spätinfekten gelang die Infektsanierung unter Prothesenerhalt nicht mehr. Nur bei 1 von 22 Patienten mit Spätinfekten konnte die Prothese belassen werden, ansonsten waren die Explantation der Prothese und die offene Revision mit temporärer Transfixierung erforderlich.

Eine Vielzahl von Risikofaktoren prädisponiert für die Entwicklung von Protheseninfektionen. Patienten mit inflammatorischen Grunderkrankungen, wie z. B. chronischer Polyarthritis, weisen ein erhöhtes Risiko auf $[1,14]$. Endogene Risikofaktoren, wie z. B. Diabetes mellitus, periphere arterio-venöse Verschlusskrankheit (pAVK) und Adipositas, und exogene Risikofaktoren, wie z. B. langjährige Kortisontherapie und Nikotinabusus, sind ebenfalls mit einem erhöhten Risiko verbunden [2, 7, 14].

Die lokalen Haut- und Weichteilverhältnisse sind für die ungestörte Wundheilung von besonderer Bedeutung. Vorausgegangene Operationen, die zu schlechteren Durchblutungsverhältnissen führen, und Knieprothesen mit unzureichender Weichteildeckung sind Risikofaktoren, aus denen sich Wundheilungsstörungen entwickeln können $[11,13]$.

Auf die Bedeutung einer Zentrumserfahrung wird in der Literatur immer wieder hingewiesen, da die Operationsdauer und die Anzahl der Personen im Operationssaal als Risikofaktoren für eine mögliche perioperative Kontamination gelten $[7,11]$. Invasive Therapie im Mund-Kiefer-Gesichts- und HNO-Bereich sowie im urologischen und gynäkologischen Bereich, die ohne Antibiotikaschutz durchgeführt werden, können über eine hämatogene Streuung zu einem Protheseninfekt führen [5].

Die Indikation zum Prothesenerhalt kann gestellt werden, wenn ein limitierter Infekt vorliegt, eine ausreichende Deckung von reizlosen Weichteilen gegeben ist, der Streckapparat und die Bewegungsfunktion erhalten sind sowie ein ausreichendes knöchernes Fundament vorhanden ist $[3,7,8,11]$. Der Patient muss nach Aufklärung das aufwändige Revisionskonzept von programmierten Lavage-Operationen mit tragen, und es muss ein entsprechender guter Allgemeinzustand vorliegen.

Als individueller Therapieansatz kann insbesondere beim alten Patienten mit Risikofaktoren ein einzeitiger Prothesenaustausch in Betracht kommen, 
wenn das zweizeitige Vorgehen aufgrund eines reduzierten Allgemeinzustands nicht indiziert ist.

Der zweizeitige Prothesenaustausch wird vorwiegend bei Spätinfekten und sanierungsbedürftigen Weichteilen [4] sowie bei jüngeren Patienten im guten Allgemeinzustand angewendet, bei denen die Compliance für ein aufwändiges Revisionskonzept vorhanden ist.

Die Behandlungsziele nach Infektion einer Knieendoprothese sind

- Infektheilung,

- Erzielen von Schmerzfreiheit,

- Funktionserhalt und

- erfolgreiche soziale Integration.

\section{Fazit für die Praxis}

Die Infektion erfordert eine frühzeitige

Diagnostik und definitive Therapie. Jede Wundheilungsstörung muss sorgfältig evaluiert und konsequent behandelt werden. Nur der Frühinfekt kann unter Prothesenerhalt saniert werden. Unter Anwendung eines konsequenten Revisionsprotokolls können die Infektheilung unter Prothesenerhalt oder eine erfolgreiche Revisionsendoprothetik gelingen.

\section{Literatur}

1. Bengtson S, Knutson K (1991) The infected knee arthroplasty. A 6-year follow-up of 357 cases. Acta Orthop Scand 62: 301-311

2. Berbari EF, Hanssen $A D$, Duffy MC, Steckelberg JM, Ilstrup DM, Harmsen WS, Osmon DR (1998) Risk factors for prosthetic joint infection: casecontrol study. Clin Infect Dis 27: 1247-1254

3. Drobny TK, Munzinger UK, Chomiak J (1995) 2-stage exchange in the treatment of infected knee prosthesis. Orthopäde 24: 360-366

4. Fansa H, Plogmeier K, Schenk K, Schneider W (1998) Covering extensive soft tissue defects in infected knee endoprostheses by gastrocnemius flap. Chirurg 69: 1238-1243

5. Fuchs S, Mersmann A (2000) Results of arthrodesis after knee prosthesis infection. Special reference to quality of life. Unfallchirurg 103: 626-631

6. Hanssen AD, Rand JA (1999) Evaluation and treatment of infection at the site of a total hip or knee arthroplasty. Instr Course Lect 48: 111-122
7. Härle A (1991) Infection of knee endoprosthesis. Orthopäde 20: 227-238

8. Insall JN, Thompson FM, Brause BD (2002) Twostage reimplantation for the salvage of infected total knee arthroplasty.J Bone Joint Surg Am 84-A: 490

9. Jansen B, Schumacher-Perdreau F, Peters $G$, Pulverer $G$ (1989) New aspects in the pathogenesis and prevention of polymer-associated foreign-body infections caused by coagulasenegative staphylococci.J Invest Surg 2: 361380

10. Johnson DP, Bannister GC (1986) The outcome of infected arthroplasty of the knee.J Bone Joint Surg Br 68: 289-291

11. Morrey BF, Westholm F, Schoifet S, Rand JA, Bryan RS (1989) Long-term results of various treatment options for infected total knee arthroplasty. Clin Orthop 248: 120-128

12. Rand JA, Morrey BF, Bryan RS (1984) Management of the infected total joint arthroplasty. Orthop Clin North Am 15: 491-504

13. Siegel A, Frommelt L, Runde W (2000) Therapy of bacterial knee joint infection by radical synovectomy and implantation of a cemented stabilized knee joint endoprosthesis. Chirurg 71: 1385-1391

14. Wilson MG, Kelley K, Thornhill TS (1990) Infection as a complication of total knee-replacement arthroplasty. Risk factors and treatment in sixty-seven cases. J Bone Joint Surg Am 72: 878-883 